\title{
Methods in Social Anthropology*
}

$\mathrm{I}^{\mathrm{N}}$ opening his lecture in memory of Thomas Henry Huxley, Prof. Westermarck said that it was calculated in a peculiar way to call to his mind the first steps he ventured to take in social anthropology nearly fifty years ago, when, being concerned with the origin of marriage, and thinking with other scholars of the time that primitive man lived in a state of promiscuity, he became acquainted with the doctrine of organic evolution and drew the conclusion that the social habits of the anthropoids might throw some light on those of primitive man. It is now to be regarded as amply proved that among many of the apes the social unit is the family, and it may be concluded that the factors which necessitated marital and paternal relations among the apes also presumably operated among our earliest human or halfhuman ancestors. He went on to point out, however, that apart from its role in the production of instinets, the principle of natural selection does not render us much help in our search for social origins.

In his study of social origins, the sociologist is much better situated than the biologist in his study of organic evolution. Natural selection presupposes organic variation, but scarcely anything is known about the origin of these variations. The causes of social phenomena, on the other hand, are to an almost unlimited degree accessible to the sociologist; and it is his business to find them. The methods of sociology, therefore, are ultimately the methods of finding the causes of social phenomena.

One method of social anthropology is the comparative one. This method starts from the fact that there are great similarities between the products of culture. The task of comparative sociology is not so much to classify these facts as to answer the question why there is this similarity. Tylor, Huxley, Frazer, have all recognized the fact that these similarities may be due to a similarity in the operations of the human mind in like conditions, or they may be due to diffusion or borrowing; and the criticisms of Rivers, Elliot Smith and others to the effect either that the possibility of diffusion is not recognized, or if it is that this involves a contradiction, are not well grounded. It is true that the question whether a certain custom or institution has sprung up inde. pendently among the people or tribe practising it, or whether it has been introduced from some other people or tribe, is seldom discussed in comparative treatises. But this by no means implies the assumption of independent origin; on the contrary, when the custom or institution occurs among related or neighbouring peoples, there is, at least in many cases, a tendency almost to take it for granted that it has been derived from a common source. Taking the study of proverbial sayings as an example, peoples have at all times taken adages from each other. But it must always be borne in mind that the resemblance between proverbs may have another cause than diffusion, namely, the people's tendency to express themselves in apophthegmatic sayings, which, owing to their terseness, are apt to become more or less similar in similar situations. The real test

- Substance of the Huxley Memorial Lecture for 1936, delivered by Prof. Edward Westermarck before the Royal Anthropological Institute on October 27. of a common origin is, therefore, not the mere similarity of ideas and sentiments revealed in the proverbs, but the similarity of formal expression, with due allow. ance for modifications that are liable to occur when a saying is adopted from another language and transplanted into a new soil.

Another branch of cultural phenomena in which diffusion has played a prominent part is decorative art. The diffusion may not in every case refer directly to particulars in the design ; it may also be an independent "effect of the idea underlying it. The patterns used in Morocco were originally to a large extent charms against the evil eye. They are based on two principles, the hand, or its five fingers, and the image of an eye or pair of eyes. Both types of charm are spread all over the Mediterranean, and many of them also farther east, and are proved to have existed since very early times. This does not preclude the possibility of more or less similar representations of protective objects having in certain instances originated independently, which is illus. trated by the occurrence of designs or charms on the leather bags of the mountaineers of Morocco in the form of a pair of eyes resembling the capital of an Ionic column. That the capital of an Ionic column may have its origin in a charm against evil protecting the temples of the Greeks would be no wonder ; but it is extremely improbable that the Moorish charms have been copied from the Ionic capital. Their resemblance is easily explained by a common belief that the representation of the eyes serves as a protection against the evil eye. Widely distributed, the eye ornament may have a different meaning in different cases, illustrating a weakness in the method of diffusionists, who have a tendency to rely on external resemblances only.

Comparative study further may enable us to discover that certain customs are vestigial forms, so called survivals a custom which cannot be explained by present circumstances, but persists in isolation from its original context. In spite of the importance of this principle, there has never been any genera theoretical discussion of its proper application, where historical evidence is absent. Two definite rules may be laid down. A custom must not be interpreted as a survival of something of which it cannot be a survival, and it must not be regarded as the survival of something, which is not known to have existed, or the earlier existence of which may not be assumed for some other reason, as for example in the so-called survivals of an ancient state of group marriage.

Nowadays the reaction against the evolutionary school of anthropologists has become so strong that any attempt to conjecture the origin of a social institution or of an element of culture has been pronounced to be out of place in social anthropology. It is, however, in this field that social anthropology has performed some of its most important tasks. Its results are to a large extent as well established as any reached in any sphere of research. The common complaint of the 'new anthropology' that the com. parative method detaches the cultural phenomenon from the organic whole, of which it forms part, is largely due to the incompleteness of the sources at the sociologist's disposal, and can be remedied by 
careful monographs on particular cultures. The 'new anthropology' aims at discovering general laws, but it is doubtful whether such general laws, if discovered, would be of much service in investigating social phenomena.

On the other hand, the view expressed by Prof. Radcliffe-Brown that "no insight, however genial, can fully compensate for the absence of direct personal contact with the kind of material that the anthropologist has to study and explain", is to be fully endorsed. It is borne out by Prof. Westermarck's personal experience of nine years close contact with the Moors, spread over more than three decades, and spent in investigating their customs and ideas.

\section{Morphogenesis and the Field Concept}

$\mathrm{T}$ HE discussion on morphogenesis which was held in Section D (Zoology) during the Blackpool meeting of the British Association was concerned mainly with the use of the field concept in experimental embryology. The central problem of the causal analysis of development is to account for the production of organs, that is to say, of masses of tissue which are integrated into units. Such integration can only be dealt with in terms of something which extends throughout the whole volume of the mass, and such extended entities are now usually referred to as 'fields'.

The first problem which arises is to give a satisfactory description of the integral behaviour. The sequence of shapes which are assumed by an organ during development has been described in great detail for most animal organs, but we require now some way of summing up, for each organ, the whole assemblage of shapes in a way which expresses the unitary character underlying the series. Probably a new branch of mathematics will be necessary to deal with such unfolding of even more complicated forms, though Dr. J. Needham mentioned the application of topological analysis and Gaussian curve analysis as hopeful possibilities. Mr. C. H. Waddington gave an example of what can already be attempted in some of the simpler cases ; the shape of the notochord and somites of the early chick embryo can be expressed as the product of two damped wave functions, with the time variable in the expression for the damping of the longitudinal wave function, so that as time proceeds more waves (that is, somites) are formed. It is possible, by causing the embryonic body to develop in a bent form, to distort the co-ordinate network in which the functions are measured, and experiments of this kind may make it possible to choose, from mathematical possibilities, the particular formulation which most immediately expresses the actual forces at work.

The integrated or organized behaviour of developing organs is shown by the regulation of parts of the organ to reconstitute the whole; such experiments make it clear that the behaviour of a part of a developing organ is a function of its position in the whole. Dr. P. D. F. Murray deseribed some beautiful examples of this type of behaviour, taken from his own earlier work on chorio-allantoic grafts of fragments of the limb-bud of chick embryos, and from Miss H. B. Fell's recent work on tissue culture of the same material. Thus if the isolated fragment contains slightly more tissue than would normally take part in the formation of the femur, it never. theless forms no more than a complete femur, which therefore incorporates the extra tissue. Dr. Murray also discussed the mosaic development of later stages in which regulation is no longer possible. Prof. E. A.
Spaul's interesting and important communication on the hormonal control of metamorphosis in Amphibia also dealt with aspocts of morphogenesis which do not come into the scope of this short note.

Regulation can only be analysed in ordinary causal terms if we suppose that the series of whole unit shapes, to which regulation occurs, is an equilibrium-series, that is, an equilibrium which is not constant but follows a definite course in time. According to this view, all the forces which at any time produce the developmental form-changes of an organ are in equilibrium with one another, and if the equilibrium is disturbed, for example, by removing part of the tissue, the same forces restore the equilibrium and thus cause the regulation of the organ. Regulation is therefore due to the interaction of forces proceeding from the different parts of the developing organ-rudiment. In so far as it is dependent on forces proceeding from different parts, it is dependent on local differences within the organ rudiment, and is in this respect similar to mosaic development, which is often and wrongly taken to be the exactly opposite type of behaviour to regula. tion. According to the present point of view, the differences between them are much less important; both types of behaviour depend upon local differences within the developing mass, but in cases where regulation occurs these differences are still labile and are capable of being altered by their mutual interaction, while in the mosaic stage the differences have become fixed and stable.

Dr. Needham concerned himself mainly with the nature of the forces which are concerned in setting up such equilibria. He directed particular attention to the possibility of finding a basis for the structural differentiation of masses of tissue in the arrangement of anisomorphic micelles or molecular aggregates. We know both that such molecules exist in protoplasm and that they can be arranged into orientated masses of the nature of liquid crystals. Such facts make it possible for the biochemist to form a picture of what it is the embryologist may be referring to when he invokes a 'cytosquellette' or an 'Intimstruktur'. The time has not yet come, he said, for an attempt at a detailed application of such ideas. We want to know, in particular, something about the maximum size of the volumes which can be affected by such molecular structures. Can we expect the orientated arrangements to be large enough to be invoked as an immediate explanation of such phenomena as the closure of the neural plate or the gastrulation movements in an amphibian egg, or must we only expect to find them active as deter. mining the positions of other agencies which are the immediate causes of the more large-scale changes of form? 